\title{
Examining potential effects of non- occupational post-exposure prophylaxis (nPEP) on sexual behaviors of Chinese men who have sex with men: a cross-sectional study
}

Haochu Li ${ }^{1 *+}$, Ran Wei ${ }^{1+}$, Jason J. Ong ${ }^{2}$, Eunsook Kim³ ${ }^{3}$ Traci L. Weinstein ${ }^{4}$, Xiaofu Ning ${ }^{5}$ and Wei Ma ${ }^{1 *}$ (D)

\begin{abstract}
Background: In China, non-occupational post-exposure prophylaxis (nPEP) is not a conventional service yet and nPEP related studies are very few. Recently, China's 13th Five Year Action Plan for HIV/AIDS Containment and Prevention examines the feasibility of including nPEP as one of the national strategies for HIV prevention. However, there is a concern that nPEP use might exacerbate high-risk sexual activities. In order to facilitate a research-based policy making of routinizing nPEP services, the current study examined potential effects of nPEP use on condom use and number of sexual partners among Chinese men who have sex with men (MSM) .
\end{abstract}

Methods: A cross-sectional survey was conducted in two cities of China in November and December 2018. Descriptive analyses of participants' sociodemographic and behavioral characteristics were conducted using SPSS 24.0. Mplus 7.4 was used to conduct confirmatory factor analysis and structural equation modeling.

Results: The sample included 419 Chinese MSM with a mean age of 28.04 (SD =9.71). Participants reported more positive anticipation of their own behaviors than other MSM's behaviors regarding condom use and number of sexual partners if nPEP were to be routinized in China. About $60 \%$ of participants reported discrepancies between anticipated individual and population behaviors as a potential result of routinization of nPEP services. Anticipated individual behavioral change was positively related to age and duration of residence in the current city, and negatively related to education. Anticipated population behavioral change was positively related to age. Anticipated behavioral discrepancy was positively related to being ethnic minority and never married.

\footnotetext{
* Correspondence: haochuli@link.cuhk.edu.hk; weima@sdu.edu.cn

${ }^{\dagger}$ Haochu Li and Ran Wei contributed equally to this work.

${ }^{1}$ Department of Epidemiology, School of Public Health, Cheeloo College of

Medicine, Shandong University, 44 West Wenhua Road, Jinan 250012,

Shandong Province, China

Full list of author information is available at the end of the article
}

(c) The Author(s). 2021 Open Access This article is licensed under a Creative Commons Attribution 4.0 International License, which permits use, sharing, adaptation, distribution and reproduction in any medium or format, as long as you give appropriate credit to the original author(s) and the source, provide a link to the Creative Commons licence, and indicate if changes were made. The images or other third party material in this article are included in the article's Creative Commons licence, unless indicated otherwise in a credit line to the material. If material is not included in the article's Creative Commons licence and your intended use is not permitted by statutory regulation or exceeds the permitted use, you will need to obtain permission directly from the copyright holder. To view a copy of this licence, visit http://creativecommons.org/licenses/by/4.0/. The Creative Commons Public Domain Dedication waiver (http://creativecommons.org/publicdomain/zero/1.0/) applies to the data made available in this article, unless otherwise stated in a credit line to the data. 
(Continued from previous page)

Conclusions: These findings identify a high-risk subgroup of MSM, who reported they would use condoms less and/or have more sexual partners when nPEP becomes available. This subgroup of MSM might benefit from targeted health interventions. Moreover, there is a clear discrepancy between anticipated individual and population behavioral changes regarding future routinization of nPEP services, suggesting incorporating nPEP services as a means of community development for MSM.

Keywords: Anticipated behavioral change, Condom use, number of sex partners, Non-occupational post-exposure prophylaxis (nPEP), Men who have sex with men, China

\section{Background}

Non-occupational post-exposure prophylaxis (nPEP) is effective in reducing human immunodeficiency virus (HIV) transmission and involves the administration of antiretroviral drugs within $72 \mathrm{~h}$ of an exposure where there is a substantial likelihood for HIV transmission and continuing the treatment for a total of 28 days [1]. nPEP is recommended when a person's sexual partner is HIV-positive with a detectable or unknown viral load or there was condomless anal sex with men who have sex with men (MSM) of unknown HIV status [1]. nPEP programs have been implemented in many resource-rich countries to complement other effective HIV prevention strategies [2]. The World Health Organization has also provided guidance on the use of nPEP [3]. Studies reported that the use of antiretrovirals after a high risk exposure can reduce the likelihood of HIV infection by $79-81 \%$ [4] and nPEP is cost-effective when targeted to high-risk populations $[5,6]$. However, there is a perception that nPEP might exacerbate high-risk sexual activities, also called sexual risk behavior disinhibition $[7,8]$. In this regard, studies to estimate potential nPEP effects on sexual behaviors among key populations, such as MSM, are warranted.

The current literature show that the relationship between nPEP and sexual risk behavior is not conclusive. Some studies confirm that disinhibition of sexual risk behavior is a concern for individuals taking nPEP. A study from the United States (US) found a significant proportion (21\%) of MSM reported condomless sex and $11 \%$ of MSM reported condomless sex with HIVpositive or HIV status unknown partners during nPEP use [9]. A longitudinal study in the Netherlands found that the majority of the HIV seroconverters reported ongoing risk behavior after nPEP prescription [10]. Another longitudinal study in Australia reported an association between nPEP use and high risk of subsequent HIV infection among MSM [11]. A retrospective study in Australia also confirmed that nPEP users had a higher number of male partners, less consistent condom use, and injecting drug use history [12]. However, there are other studies that reported different results. A study in San Francisco reported that knowledge about the availability of nPEP did not lead to an increase in condomless anal sex among gay men [13]. Another longitudinal study also reported that most MSM (73\%) had a decrease in high-risk sexual behaviors and most of them (85\%) had no change in incident sexually transmitted infections (STI), indicating a lack of behavioral disinhibition [14]. Another randomized HIV-prevention trial among MSM in the US observed that, although nPEP users were a riskier group at baseline, previous nPEP use was not associated with higher odds of high risk sex at follow-up [15]. In Brazil, a cohort study also reported that nPEP use was not associated with increases in reported high-risk behavior [4]. These discrepancies require further exploration, which is a significant contribution of the current study.

Recently, China's 13th Five-Year Action Plan for HIV/ AIDS Containment and Prevention examined the feasibility of including nPEP as one of the strategies for HIV prevention. Yet, in China, there are few nPEP studies to date. One study published in a Chinese journal explored factors related to the need of nPEP and reported only $22.1 \%$ of MSM had heard about nPEP [16]. Another study described the introduction of nPEP services in an acquired immune deficiency syndrome (AIDS) designated hospital in Beijing, but did not report any impact of nPEP use on sexual risk behaviors [17]. In the English literature, nPEP studies from China are largely absent. This might be because nPEP is not a conventional service widely available in China yet, although some AIDSdesignated hospitals provide nPEP on a case-by-case basis. Before nPEP is routinized nationwide, it is necessary to examine the potential for nPEP use to change sexually risky behaviors. The current study aims to explore the potential effects of nPEP use on individual and population sexual behaviors from the perspective of MSM if nPEP is routinized nationally.

The current study is guided by social cognitive theory, which conceptualizes an interactional causal structure as triadic reciprocal causation of behavior that involves a dynamic interplay among personal determinants, behavior and environmental influences [18]. Therefore, we modelled the relationships among personal determinants (e.g., age, education, HIV knowledge, nPEP knowledge), 
behavior (e.g., using nPEP, looking for male sexual partners through the Internet), and environmental factors (e.g., city of residence, duration of local residence in the current city) in the current study. We hypothesize that nPEP use and related knowledge might be related to MSM's sexual risk behavior. Moreover, previous studies reported that nPEP use was associated with inconsistent condom use [12] and more sexual partners among MSM [19], supporting our hypothesis that MSM's behaviors regarding condom use and number of sexual partners might change if nPEP is widely available. A study has reported a significant discrepancy between people's actual risk behavior and their perception [20]. However, there are very few HIV studies examining anticipated behavioral discrepancy between individual and population. We adopted this concept from studies that examined perceived/anticipated behavioral or cognitive/emotion discrepancy in other health-related models [21, 22]. We hypothesize that nPEP use might influence nPEP knowledge, and it might influence anticipated individual and population behavioral changes and discrepancy directly and indirectly. People generally first learn about HIV, and then nPEP [23], and HIV and nPEP knowledge were associated with an intention to use nPEP [24]. In this regard, we hypothesize that HIV knowledge might have an effect on nPEP knowledge, and it might influence anticipated individual and population behavioral changes and discrepancy directly and indirectly.

\section{Methods}

A cross-sectional study that explored Chinese MSM's awareness, knowledge, and perceived need of nPEP, willingness to use nPEP, and sociodemographic factors was conducted in November and December, 2018. Two big cities in China, Shijiazhuang in the north and Xiamen in the south, were selected given that MSM populations are large and accessible in these two cities, and local centers for disease control and prevention and community-based organizations (CBOs) were able to help with the data collection. Local CBOs helped recruit participants. Inclusion criteria were: (1) biological males (assigned male sex at birth) aged 18 years and over; (2) had sex with another male in the past 12 months; (3) self-reported negative or were unaware of their HIV status; and (4) provided written informed consent and participated voluntarily in the survey. Exclusion criteria were: (1) self-reported HIV positive; (2) not able to complete the survey due to health problems (e.g., mental illness); and (3) unable to provide written informed consent.

\section{Survey measures}

The structured survey instrument was iteratively developed by first referring to China's HIV/AIDS sentinel surveillance questionnaire [25] and a scoping review of both domestic and foreign literature, revising the questionnaire according to the results of qualitative work, and consulting experts who have research or clinical experiences related to HIV and nPEP among MSM. The instrument covered topics such as socio-demographic information, HIV/AIDS knowledge, HIV-related behaviors (i.e., sexual behaviors, alcohol and drug use, and HIV testing), awareness of and need for nPEP, and potential impact of nPEP services on future sexual risk behaviors [26].

\section{HIV knowledge}

A scale with eight categorical items with three response options was used [25]. An example question is "Will infections of other sexually transmitted diseases increase the risk of getting HIV infection?" with answers of "Yes (1)", "No (0)", and "unknown (0)". Responses were transformed into correct or incorrect binary items, treating unknown as incorrect in data analysis. Higher scores indicated better knowledge of HIV/AIDS. Latent variable modeling showed that the HIV Knowledge Scale's reliability was 0.824 with $95 \%$ confidence interval $(\mathrm{CI})$ of $0.796-0.853$.

\section{nPEP knowledge}

An author-derived scale with nine items was used. An example question was "A person needs to make sure they are not infected with HIV before taking nPEP" with answers of "correct (1)", "wrong (0)", and "unknown (0)". Higher scores indicated better knowledge of nPEP. Latent variable modeling showed that the nPEP Knowledge Scale's reliability was 0.903 with $95 \%$ CI of $0.891-0.915$.

\section{HIV-related behaviors and utilization of services}

Items assessing behaviors related to HIV risk and testing included number of instances of anal sex with males in the last week, number of male sexual partners in the last 6 months, condom use in the most recent anal sex with other males in the last 6 months, condom breakage, slippage or intentional removal when having sex with other males in the last 6 months, use of illicit drugs, frequency of drinking alcohol in the last 3 months, diagnosis of sexually transmitted disease (STD) in the past 12 months, frequency of HIV testing, and use of nPEP ever.

\section{Socio-demographic information}

Nine items assessed participants' socio-demographic characteristics. Participants indicated their age, city of residence, duration of residence in the current city, education level, monthly income, marital status with women, sexual orientation, locations for looking for male sexual partners, and status of being a student. 


\section{Anticipated individual behavioral changes}

Two author-derived categorical items were used, asking for participants' opinions about future condom use behavior and number of sex partners regarding themselves. An example question is "If nPEP is promoted nationally, what change regarding your personal behavior of condom use will you have?" The answer was given as either decrease (1), no change (2), or increase (3). A higher score indicated higher positive impact of nPEP services on future risky behaviors.

\section{Anticipated population behavioral changes}

Two author-derived categorical items were used, asking for participants' opinions about future condom use behavior and number of sex partners regarding the whole MSM population. An example question is "If nPEP is promoted nationally, what change regarding MSM population's behavior of condom use will there be?" The answer was either given as decrease (1), no change (2), or increase (3). A higher score indicated higher positive impact of nPEP services on MSM population future risky behaviors.

\section{Anticipated behavioral discrepancy}

This variable was adopted and adjusted from other studies [20-22]. This was calculated as the sum of absolute values of anticipated individual change minus anticipated population change in condom use and the number of sexual partners respectively. Higher scores indicated larger discrepancy between anticipated individual and population behavior in condom use and number of sexual partners.

\section{Survey procedures}

Before conducting the survey, investigators were trained to ensure they were familiar with the survey process and understood the content of the questionnaire. A convenience sampling method was used. Local CBOs invited potential participants when they came for HIV testing and organized volunteers to invite participants from MSM venues (e.g., gay saunas). After participants provided written informed consent, they received a questionnaire link from Wenjuanxing (a professional electronic survey platform) and personal keys, which ensured better authenticity of survey results. Participants completed the survey independently on their smart phone or computer. After the submission of the questionnaire, an audit was conducted by the research team, after which participants were provided 30 Yuan, equal to United States dollar (USD) 4.50, through Wechat Red Pack as compensation. A total of 423 questionnaires were received, and 419 of them were retained after four questionnaires were deleted due to a large number of questions unanswered.

\section{Statistical analysis}

Software package for statistical analysis (SPSS) 24.0 was used to conduct a descriptive analysis of participants' sociodemographic and behavioral characteristics. Mplus 7.4 was used to conduct confirmatory factor analysis (CFA) for measurement models and structural equation modeling (SEM) for estimation of research outcomes. Goodness-of-fit of the models was assessed using the comparative fit index (CFI), the Tucker-Lewis index (TLI), and the root mean square error of approximation (RMSEA). Acceptable model fit is determined by a RMSEA less than 0.08, and values of CFI and TLI greater than 0.90 [27, 28]. The CFA solutions were evaluated with acceptable overall goodness-of-fit, no focal areas of ill fit (absence of large modification indices and standardized residuals), and no out-of-range values in the parameter estimates [29]. Latent variable modeling was used to test the scale's reliability [30]. Finally, SEM were conducted using anticipated behavioral changes and anticipated behavioral discrepancy as outcome variables respectively to estimate their relationships with nPEP knowledge, HIV knowledge, and sociodemographic and behavioral covariates (so called, a path model). We modelled nPEP knowledge as a mediator and tested the nPEP use and HIV knowledge effects on each outcome directly and indirectly through nPEP knowledge. In addition, the measurement models of nPEP knowledge, HIV knowledge, and each outcome variable were directly included in SEM (namely, structural regression model) to take into account measurement errors.

The structural regression model in SEM is a combination of measurement model (or CFA) and path model and allows researchers to model not only complex relations between variables such as mediation but also measurement errors explicitly [31-33]. Thus, researchers can conduct factor analysis and test research hypotheses simultaneously and obtain measurement-error-free estimates. For the estimation of CFA and SEM, we used weighted least squares with mean and variance adjusted (WLSMV) instead of commonly used maximum likelihood (ML) because the outcome variables in this study were categorical. Because multivariate normality cannot be assumed with categorical variables, the WLSMV is considered an appropriate estimator and the outperformance of WLSMV over ML was reported for categorical data when the number of categories was less than five [34-36].

\section{Results}

\section{Sociodemographic and behavioral characteristics}

The sample included 419 participants with a mean age of 28.04, with a standardized deviation (SD) of 9.71. The majority (78.8\%) were aged between 18 and 34 years. 
Table 1 Sociodemographic and behavioral characteristics of MSM in two cities of China, 2018 ( $N=419)$

\begin{tabular}{|c|c|c|}
\hline & Number & Percent \\
\hline \multicolumn{3}{|l|}{ Age $($ mean $=28.04, \mathrm{SD}=9.71$, years $)$} \\
\hline $18-34$ & 330 & 78.8 \\
\hline $35-49$ & 69 & 16.5 \\
\hline $50-64$ & 19 & 4.5 \\
\hline $65+$ & 1 & 0.2 \\
\hline \multicolumn{3}{|l|}{ City of residence } \\
\hline North & 217 & 51.8 \\
\hline South & 202 & 48.2 \\
\hline \multicolumn{3}{|l|}{ Ethnicity } \\
\hline Han & 407 & 97.1 \\
\hline Non-Han (other ethnicity) & 12 & 2.9 \\
\hline \multicolumn{3}{|c|}{ Duration of residence in the current city } \\
\hline$<3$ months & 22 & 5.3 \\
\hline $3-6$ months & 22 & 5.3 \\
\hline 7-12 months & 20 & 4.8 \\
\hline $13-24$ months & 60 & 14.3 \\
\hline$>24$ months & 295 & 70.4 \\
\hline \multicolumn{3}{|l|}{ Education } \\
\hline Illiterate & 4 & 1.0 \\
\hline Primary school & 5 & 1.2 \\
\hline Secondary school & 47 & 11.2 \\
\hline High/Polytechnic school & 87 & 20.8 \\
\hline College or above & 276 & 65.9 \\
\hline \multicolumn{3}{|l|}{ Monthly income $(\mathrm{CYN})^{\text {a }}$} \\
\hline$<1500$ & 89 & 21.2 \\
\hline $1500-3000$ & 72 & 17.2 \\
\hline $3001-5000$ & 123 & 29.4 \\
\hline $5001-8000$ & 75 & 17.9 \\
\hline$>8000$ & 49 & 11.7 \\
\hline Missing & 11 & 2.6 \\
\hline \multicolumn{3}{|l|}{ Marital status with female } \\
\hline Never married & 322 & 76.8 \\
\hline Married & 68 & 16.2 \\
\hline Cohabiting & 13 & 3.1 \\
\hline Divorced/separated/widowed & 16 & 3.8 \\
\hline \multicolumn{3}{|l|}{ Sexual orientation } \\
\hline Homosexual & 260 & 62.1 \\
\hline Bisexual & 106 & 25.3 \\
\hline Heterosexual & 16 & 3.8 \\
\hline Uncertain & 37 & 8.8 \\
\hline \multicolumn{3}{|c|}{ Number of episodes of anal sex with males in the last week } \\
\hline 0 & 245 & 58.5 \\
\hline $1-4$ & 162 & 38.7 \\
\hline $5-8$ & 10 & 2.4 \\
\hline
\end{tabular}


Table 1 Sociodemographic and behavioral characteristics of MSM in two cities of China, 2018 ( $N=419)$ (Continued)

\begin{tabular}{|c|c|c|}
\hline & Number & Perc \\
\hline$>8$ & 2 & 0.5 \\
\hline \multicolumn{3}{|c|}{ Number of male sexual partners in the last 6 months } \\
\hline 0 & 127 & 30.3 \\
\hline 1 & 121 & 28.9 \\
\hline $2-6$ & 146 & 34.8 \\
\hline$>6$ & 25 & 6.0 \\
\hline \multicolumn{3}{|c|}{ Condom use in the most recent anal sex with other males in the last 6 months } \\
\hline Yes & 241 & 57.5 \\
\hline No & 51 & 12.2 \\
\hline No anal sex & 127 & \\
\hline
\end{tabular}

Condom breakage, slippage or intentional removal when having sex with other males in the last 6 months

Yes 23

No 253

60.4

Unknown

16

3.8

No anal sex

Use of illicit drugs

Yes

No

Frequency of drinking alcohol in the last 3 months

0

1-2 times in the last 3 months

1-3 times per month

1-4 times per week

$>4$ times per week

Diagnosis of STD in the past 12 months

Yes

No

Frequency of HIV testing

Never

Tested more than 12 months ago

Tested once in the last 12 months

Ever used NPEP

Yes

No

Locations for looking for male sexual partners

Venues (bars, clubs, parks, and saunas

Others

Current student

Anticipated behavioral discrepancy ${ }^{\mathrm{b}}$ 
Table 1 Sociodemographic and behavioral characteristics of MSM in two cities of China, $2018(N=419)$ (Continued)

\begin{tabular}{lll}
\hline & Number & Percent \\
\hline 1 & 126 & 30.1 \\
2 & 95 & 22.7 \\
3 & 22 & 5.2 \\
4 (the highest discrepancy) & 6 & 1.4 \\
\hline
\end{tabular}

Note. ${ }^{a}$ One CYN was about 0.1486 USD. ${ }^{b}$ Anticipated behavioral discrepancy was calculated as the sum of absolute values of anticipated individual change minus anticipated population change in condom use and the number of sexual partners respectively. Higher scores indicate larger discrepancy between anticipated individual and population behavior in condom use and number of sexual partners as a whole

Most (97.1\%) were Han Chinese, and most (84.7\%) lived in their current cities for more than 12 months. A majority $(65.9 \%)$ had education of college or above, and a majority (67.8\%) had monthly incomes of China Yuan (CYN) 5000 (about USD 743) or lower. Most (76.8\%) never got married or cohabited with females. The majority (58.5\%) did not have anal sex with males in the last week, while a significant proportion (38.7\%) reported one to four episodes of anal sex and a small proportion (2.9\%) were very sexually active (more than four times in the last week). Most (64.9\%) drank alcohol in the last 3 months. Most participants (69.7\%) reported testing for HIV in the last 12 months. Only $3.6 \%$ of participants reported use of nPEP ever. About $60 \%$ of participants reported discrepancies between anticipated individual and population behavioral change (see Table 1).

Participants reported different perceptions about their own behaviors and other MSM's behaviors if nPEP services are routinized. In terms of condom use, 9.8\% of participants anticipated a decrease of condom use for themselves, while a third (34.2\%) anticipated reduced condom use for other MSM. In terms of number of sexual partners, $16.0 \%$ of participants reported increased number of sexual partners for themselves, while more than half (54.2\%) of participants anticipated increased number of sexual partners for other MSM. Correlation analyses found that the four variables were mutually and significantly correlated (see Table 2).

\section{Measurement models of HIV knowledge and nPEP knowledge}

For HIV knowledge, CFA showed that all items loaded significantly on their corresponding factor, with standardized factor loading ranging from 0.483 to 0.905 and $p<0.001$. The goodness-of-fit of the measurement model was good $(\mathrm{CFI}=0.970$, TLI $=0.958$, RMSEA $=0.043,90 \%$ CI $0.017-$ 0.065). For nPEP knowledge, CFA showed that all items loaded significantly on their corresponding factors, with standardized factor loading ranging from 0.731 to 0.999 and $p<$ 0.001 (see Table 3). The goodness-of-fit of the measurement model was acceptable $(\mathrm{CFI}=0.998$, TLI $=0.997$, RMSEA $=$ 0.056, 90\% CI 0.038-0.074).

\section{Structural equation model of anticipated individual behavioral changes with nPEP knowledge, HIV knowledge, and covariates among Chinese MSM}

For anticipated behavioral changes on individual MSM, the structural equation model (see Fig. 1) indicated good fit indexes (RMSEA $=0.013,90 \%$ CI $0.000-0.021$, CFI $=0.995$, $\mathrm{TLI}=0.994)$. Anticipated individual behavioral changes were was not significantly related to the use of nPEP $(b=-0.101$, $p=0.185)$, HIV knowledge ( $\mathrm{b}=0.037, p=0.782)$, or nPEP knowledge $(b=0.140, p=0.232)$. Instead, they were associated with age $(\mathrm{b}=0.333, p<0.05)$, duration of residence in the current city $(\mathrm{b}=0.172, p<0.05)$, and education $(\mathrm{b}=-$ $0.251, p<0.01$ ). Anticipated individual behavioral changes were not significantly related to the use of nPEP $(b=-$ $0.101, p=0.185)$, HIV knowledge $(b=0.037, p=0.782)$, or nPEP knowledge $(\mathrm{b}=0.140, p=0.232)$. nPEP knowledge was associated with HIV knowledge, age, and use of PEP. HIV knowledge was associated with age, city of residence, never getting married, and mainly looking for sexual partners through the Internet.

Table 2 Individual and population differences of anticipated changes on condom use and number of sexual partners among Chinese MSM if NPEP services are routinized, $2018(N=419)$

\begin{tabular}{llllll}
\hline & \multicolumn{2}{l}{ Condom use } & & & \multicolumn{2}{l}{ Number of sexual partners } \\
\cline { 2 - 3 } & Individual & Population & & Individual & Population \\
\hline Increase & $40.3 \%(169 / 419)$ & $38.4 \%(161 / 419)$ & & $16.0 \%(67 / 419)$ & $30.2 \%(227 / 419)$ \\
No change & $49.9 \%(209 / 419)$ & $27.4 \%(115 / 419)$ & & $30.1 \%(126 / 419)$ \\
Decrease & $9.8 \%(41 / 419)$ & $34.2 \%(143 / 419)$ & & $17.2 \%(72 / 419)$ & $15.7 \%(66 / 419)$ \\
\hline
\end{tabular}

Note. Correlation analyses found that the four variables were mutually and significantly correlated: anticipated individual condom use with anticipated individual number of sexual partners ( $r h o=0.207, p<0.001$ ), anticipated population condom use with anticipated population number of sexual partners (rho $=0.307, p<$ 0.001 ), anticipated individual condom use with anticipated population condom use (rho $=0.568, p<0.001$ ), anticipated individual number of sexual partners with anticipated population number of sexual partners ( $r h o=0.465, p<0.001$ ), anticipated individual condom use with anticipated population number of sexual partners ( $r$ o $=0.163, p=0.001$ ), anticipated individual number of sexual partners with anticipated population condom use (rho $=0.186, p<0.001$ ) 
Table 3 Unstandardized and standardized loading for measurement models of HIV knowledge scale and NPEP knowledge scale among Chinese MSM, 2018 ( $N=419)$

\begin{tabular}{|c|c|c|}
\hline Parameter estimate & Unstandardized loading (SE) & Standardized loading $(S E)$ \\
\hline \multicolumn{3}{|c|}{$\begin{array}{l}\text { HIV Knowledge Scale confirmatory factor analysis model } \\
\text { Fit indices: CFI }=0.970, T L I=0.958, \text { RMSEA }=0.043,90 \% \text { Cl: } 0.017-0.065\end{array}$} \\
\hline $\mathrm{HIV} \rightarrow \mathrm{B} 1$ & 1.000 & $0.483(0.080)^{* * *}$ \\
\hline $\mathrm{HIV} \rightarrow \mathrm{B} 2$ & $1.136(0.219)^{* * *}$ & $0.548(0.075)^{* * *}$ \\
\hline $\mathrm{HIV} \rightarrow \mathrm{B} 3$ & $1.208(0.261)^{* * *}$ & $0.583(0.074)^{* * *}$ \\
\hline $\mathrm{HIV} \rightarrow \mathrm{B} 4$ & $1.321(0.248)^{* * *}$ & $0.638(0.058)^{* * *}$ \\
\hline $\mathrm{HIV} \rightarrow \mathrm{B} 5$ & $1.711(0.329)^{* * *}$ & $0.826(0.090)^{* * *}$ \\
\hline $\mathrm{HIV} \rightarrow \mathrm{B} 6$ & $1.667(0.290)^{* * *}$ & $0.805(0.051)^{* * *}$ \\
\hline $\mathrm{HIV} \rightarrow \mathrm{B} 7$ & $1.874(0.316)^{* * *}$ & $0.905(0.051)^{* * *}$ \\
\hline $\mathrm{HIV} \rightarrow \mathrm{B} 8$ & $1.366(0.271)^{* * *}$ & $0.659(0.074) * * *$ \\
\hline \multicolumn{3}{|c|}{$\begin{array}{l}\text { NPEP Knowledge Scale confirmatory factor analysis model } \\
\text { Fit indices: CFI }=0.998, T L I=0.997, \text { RMSEA }=0.056,90 \% \mathrm{Cl} 0.038-0.074\end{array}$} \\
\hline $\mathrm{NPEP} \rightarrow \mathrm{A}$ & 1.000 & $0.875(0.019)^{* * *}$ \\
\hline $\mathrm{NPEP} \rightarrow \mathrm{B}$ & $1.102(0.025)^{* * *}$ & $0.965(0.013)^{* * *}$ \\
\hline $\mathrm{NPEP} \rightarrow \mathrm{D}$ & $1.141(0.029) * * *$ & $0.999(0.014) * * *$ \\
\hline $\mathrm{NPEP} \rightarrow \mathrm{E}$ & $0.835(0.036)^{* * *}$ & $0.731(0.032)^{* * *}$ \\
\hline $\mathrm{NPEP} \rightarrow \mathrm{F}$ & $0.988(0.028)^{* * *}$ & $0.865(0.022)^{* * *}$ \\
\hline $\mathrm{NPEP} \rightarrow \mathrm{G}$ & $0.874(0.035)^{* * *}$ & $0.765(0.030)^{* * *}$ \\
\hline $\mathrm{NPEP} \rightarrow \mathrm{H}$ & $1.041(0.023) * * *$ & $0.911(0.016)^{* * *}$ \\
\hline $\mathrm{NPEP} \rightarrow \mathrm{I}$ & $1.107(0.026) * * *$ & $0.969(0.013) * * *$ \\
\hline $\mathrm{NPEP} \rightarrow \mathrm{K}$ & $0.905(0.032)^{* * *}$ & $0.793(0.026)^{* * *}$ \\
\hline
\end{tabular}

Note. As suggested by the modification indices, adjustment of the confirmatory factor analysis model is not needed. Standard errors are in the parenthesis. ${ }^{*} p<$ $0.05 * *<0.01 * * 0<0.001$

\section{Structural equation model of anticipated population behavioral changes with nPEP knowledge, HIV knowledge, and covariates among Chinese MSM}

For anticipated behavioral changes on MSM population, the structural equation model (see Fig. 2) indicated good fit indexes $(\mathrm{RMSEA}=0.012,90 \%$ CI $0.000-0.021$, CFI = 0.996 , TLI $=0.995)$. Anticipated population behavioral changes were was not significantly related to the use of nPEP $(b=0.020, p=0.730)$, HIV knowledge $(b=-0.005$, $p=0.965)$, or nPEP knowledge $(\mathrm{b}=-0.070, p=0.472)$. Instead, they were only associated with age $(b=0.191$, $p$ < 0.05). Anticipated population behavioral changes were not significantly related to the use of nPEP $(b=$ $0.02, p=0.73)$, HIV knowledge $(b=-0.005, p=0.965)$, or nPEP knowledge $(\mathrm{b}=-0.07, p=0.472)$. nPEP knowledge was associated with HIV knowledge, age, and use of nPEP ever. HIV knowledge was associated with age, city of residence, never getting married, and looking for sexual partners through the Internet mainly.

\section{Structural equation model of anticipated behavioral discrepancy with nPEP knowledge, HIV knowledge, and covariates among Chinese MSM}

The structural equation model (see Fig. 3) indicated good fit indexes $(\mathrm{RMSEA}=0.014,90 \%$ CI $0.000-0.023$,
$\mathrm{CFI}=0.994$, TLI $=0.993)$. Anticipated behavioral discrepancy was associated with being non-Han Chinese $(\mathrm{b}=0.105, p<0.05)$, and never getting married $(\mathrm{b}=$ $0.178, p<0.05)$. Anticipated behavioral discrepancy was not significantly related to the use of nPEP $(b=-0.055$, $p=0.332)$, HIV knowledge $(\mathrm{b}=0.065, p=0.456)$, or nPEP knowledge $(b=-0.003, p=0.965)$. nPEP knowledge was associated with HIV knowledge, age, and use of nPEP ever. HIV knowledge was associated with age, city of residence, never getting married, and mainly looking for sexual partners through the Internet.

\section{Discussion}

To the best of our knowledge, this is the first study from China to explore anticipated behavioral change in terms of condom use and number of sexual partners from MSM's perspective if nPEP were to be provided nationally. We found a significant proportion (50\%) of participants reported their sexual behaviors may change. About $60 \%$ reported anticipated discrepancies between their personal behaviors and other men's behaviors. nPEP use as well as HIV and nPEP knowledge were not significantly related to the anticipation of individual and population behavioral changes and discrepancy regarding condom use and number of sexual partners, which is consistent with 


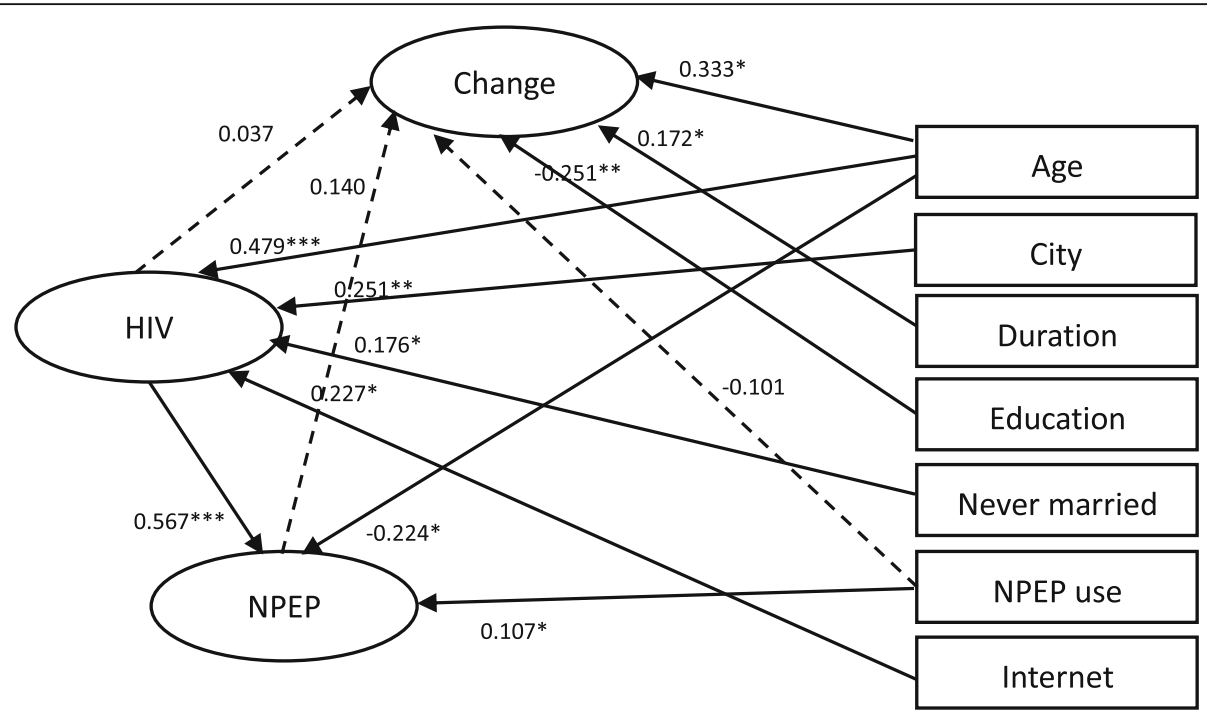

Fig. 1 Structural equation model of anticipated individual behavioral change with HIV knowledge, NPEP knowledge and covariates among Chinese MSM $(N=419)$. Note. ${ }^{*} p<0.05,{ }^{*} p<0.01,{ }^{* *} p<0.001$; Fit index: RMSEA $=0.013,90 \% \mathrm{Cl} 0.000-0.021, \mathrm{CFI}=0.995, \mathrm{TLI}=0.994 ; \mathrm{HIV}=\mathrm{HIV}$ knowledge, Change $=$ anticipated individual behavioral change, NPEP $=$ NPEP knowledge, Age $=$ age, City $=$ city of residence, Duration $=$ duration of local residence in the current city, Education = level of education, Never married = never married, NPEP use $=$ the use of NPEP, Internet $=$ mainly looking for male sexual partners through the Internet. The full model estimated the relationships among anticipated individual behavioral change (the outcome), HIV knowledge, NPEP knowledge, and all covariates, but only significant paths were shown in solid lines for simplicity. Dotted lines were the primary paths of interest but not statistically significant. All path coefficients shown were standardized. The oval represents a latent construct measured by multiple items which are not shown in the diagram for simplicity

previous studies reporting that nPEP would not lead to sexual risk behavior disinhibition $[4,13-15]$.

Our study has found some factors related to the anticipation of individual and population behavioral changes. Age is an important factor, since older men were more likely to state they will use more condoms and have fewer sexual partners if nPEP is available. It is reasonable to assume that as these men get older, they may have more social and sexual experiences regarding condom use and sexual partners. As HIV/AIDS prevention and intervention programs continue to focus on key groups of men who have sex with men, these men are more likely to be exposed to health promotion messages regarding consistent condom use and reducing number of sexual partners, and are therefore more likely to perceive positive (i.e., less risky) behavioral changes [37, 38]. Also, when these men have longer duration of residence in these large metropolitan cities with comparatively more complex MSM networks and more likelihood to be exposed to HIV/AIDS campaigns and messages, they might be more likely to understand the importance of using more condoms and having fewer sexual partners. Participants with higher education were more likely to state they would use less condoms and have more sexual partners if nPEP is available, indicating that these well-educated MSM might be overly optimistic about the protective effect of nPEP. These findings are different from a study conducted in the US reporting no significant differences in age or education level between those who reported condomless anal sex during nPEP and those who did not [9]. HIV/AIDS treatment optimism may be a concern among Chinese MSM, particularly those who are well-educated [13]. This is consistent with foreign studies reporting a higher incidence of sexually transmitted diseases was associated with perceiving less HIV/AIDS threat since highly active antiretroviral therapy availability [39]. A longitudinal study reported that nPEP was more often prescribed for a sexual accident with a known HIV-positive sex partner in a later period, indicating treatment optimism is a concern (41.0 versus $24.5 \%, P<0.01$ ) [10].

Another interesting finding is that there are differences of anticipated behavioral changes in condom use and number of sexual partners between what men anticipate for themselves and how they believe other men would respond to the availability of nPEP. These men seem to have more positive anticipations about themselves, with more negative anticipations about how their peers in the wider MSM community would react to the availability of nPEP. In other words, these men may not trust their peers, which might be about an issue of stigma related to same-sex sexuality (e.g., spoiled identity) among these marginalized and stigmatized sexual minorities [40]. This is consistent with an ethnographic study among Chinese MSM reporting men internalized a stigma that MSM are bad and dangerous and MSM communities are disappointing and hopeless [41]. Participants whose 


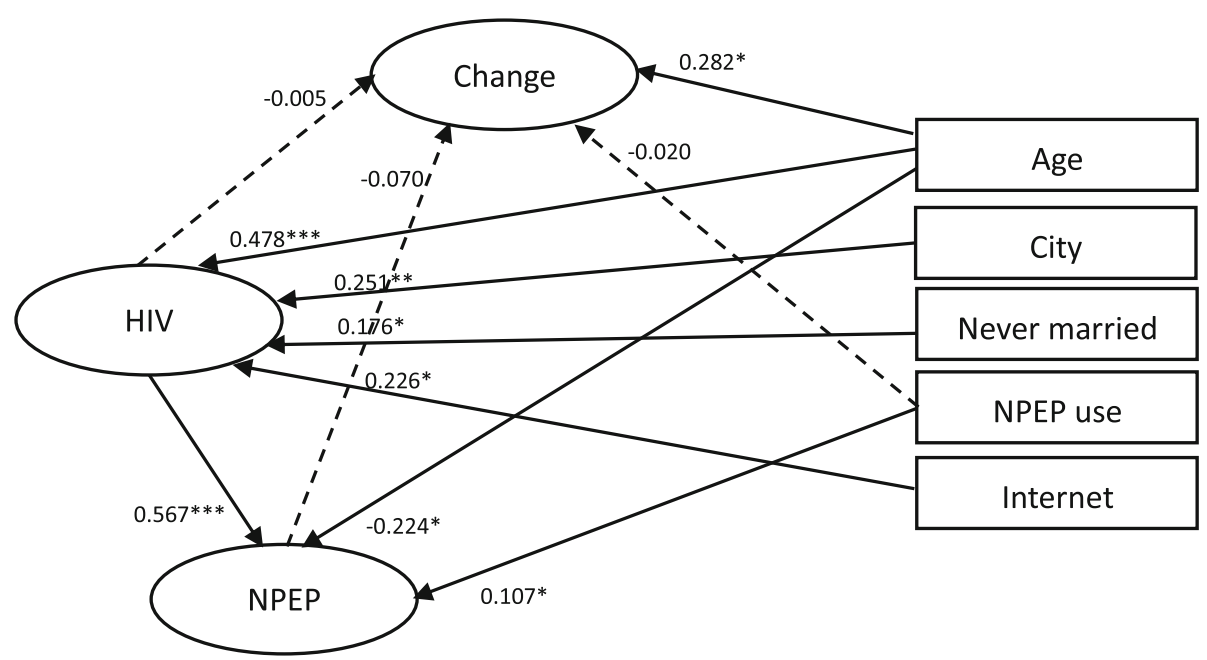

Fig. 2 Structural equation model of anticipated population behavioral change with HIV knowledge, NPEP Knowledge and covariates among Chinese MSM $(n=419)$. Note. ${ }^{*} p<0.05$, ${ }^{* *} p<0.01$, *** $p<0.001$; Fit index: RMSEA $=0.012,90 \% \mathrm{Cl} 0.000-0.021, \mathrm{CFI}=0.996, \mathrm{TLI}=0.995 ; \mathrm{HIV}=\mathrm{HIV}$ knowledge, Change $=$ anticipated population behavioral change, NPEP $=$ NPEP knowledge, Age $=$ age, City $=$ city of residence, Never married $=$ never married, NPEP use = the use of NPEP, Internet = mainly looking for male sexual partners through the Internet. The full model estimated the relationships among anticipated population behavioral change (the outcome), HIV knowledge, NPEP knowledge, and all covariates, but only significant paths were shown in solid lines for simplicity. Dotted lines were the primary paths of interest but not statistically significant. All path coefficients shown were standardized. The oval represents a latent construct measured by multiple items which are not shown in the diagram for simplicity

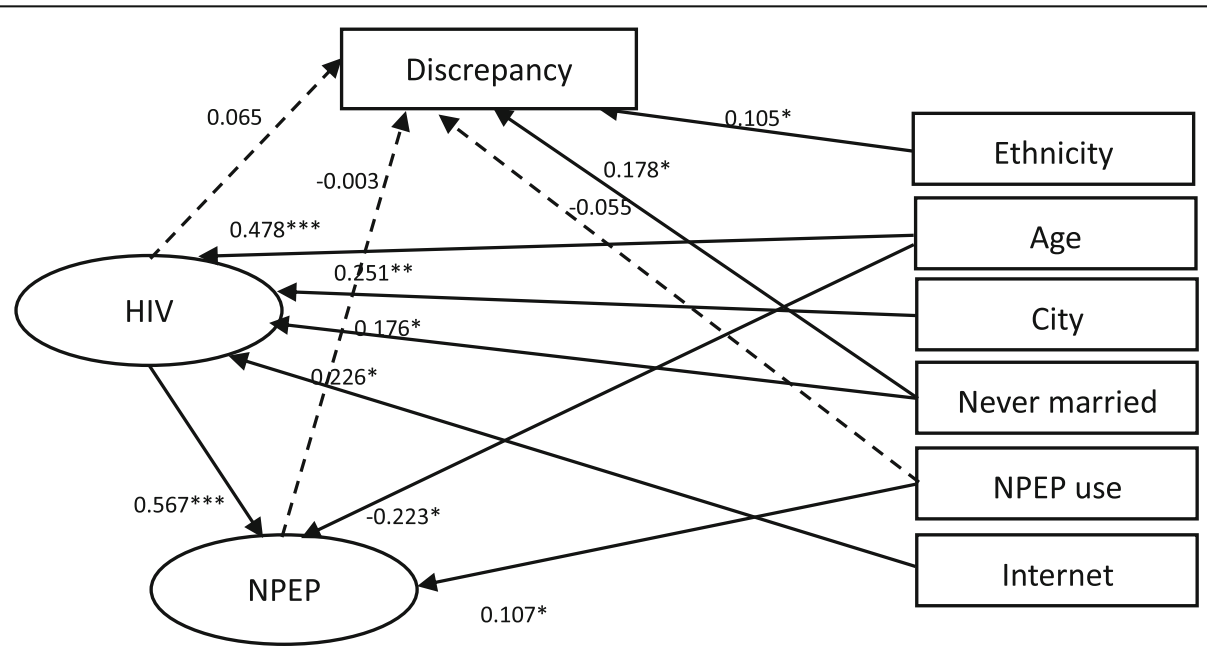

Fig. 3 Structural Model of anticipated behavioral discrepancy with HIV knowledge, PEP knowledge, and covariates among Chinese MSM ( $n=$ 408). Note. ${ }^{*} p<0.05,{ }^{* *} p<0.01,{ }^{* * *} p<0.001$; Fit index: RMSEA $=0.014,90 \% \mathrm{Cl} 0.000-0.023, \mathrm{CFI}=0.994, \mathrm{TLI}=0.993 ; \mathrm{HIV}=$ HIV knowledge, Discrepancy = anticipated behavioral discrepancy, NPEP = NPEP knowledge, Ethnicity = being non-Han Chinese, Age = age, City $=$ city of residence, Never married = never married, NPEP use = the use of NPEP, Internet = mainly looking for male sexual partners through the Internet. The full model estimated the relationships among anticipated behavioral discrepancy (the outcome), HIV knowledge, NPEP knowledge, and all covariates, but only significant paths were shown in solid lines for simplicity. Dotted lines were the primary paths of interest but not statistically significant. All path coefficients shown were standardized. The oval represents a latent construct measured by multiple items which are not shown in the diagram for simplicity 
ethnicity was not Han Chinese and participants who were never married to or cohabiting with a woman were more likely to report a discrepancy between anticipated individual and population behavioral changes. Being both an ethnic minority and an MSM may make these men even more distrustful toward their MSM peers in these metropolitan cities. For MSM who never get married and never cohabit with females, they may be more comfortable with their same-sex sexuality, which is a marginalized sexuality in China. A study reported that some MSM in metropolitan areas have tried to negotiate same-sex sexuality as a normal variation of human sexuality [42]. This practice of changing social norms is difficult, as another study reported that most (70\%) MSM got married to females after the age of 30 [43]. It is therefore understandable that MSM who never get married and never cohabit with females might have more negative perceptions about the MSM population.

Our study has important public health implications. The real use of nPEP was not significantly associated with anticipated behavioral changes, indicating that providing nPEP to MSM may not lead to their negative behavioral change regarding less condom use and more sexual partners. HIV and nPEP knowledge were not related to anticipated behavioral changes from the individual or population perspectives, indicating that health behavior promotion for increasing condom use and reducing number of sexual partners cannot just rely on distribution of knowledge. Most participants reported discrepancies between anticipated individual and population behavioral changes, suggesting distrust and internalized stigma among the MSM communities. It is important to be reflective about previous HIV/AIDS prevention and intervention projects, which largely focused on technical issues, such as testing tools and treatment regimens, with much less attention paid to the active and positive development of the communities from the perspective of the key populations. In this regard, when $\mathrm{nPEP}$ is incorporated into public health services, it needs to be implemented as a means of community development for MSM. For example, community-based MSM organizations and related stakeholders should be actively recruited to play important roles in the development and implementation of nPEP services at every step.

The current study is subject to limitations. First, the use of convenience sampling and relatively small sample size would limit the generalizability of the findings. Second, regarding the outcomes of anticipated behavioral changes, these are self-reported data and social desirability may be a concern, although this may be mitigated by the anonymous nature of the survey. MSM-related stigma may not be the only potential cause of these men's anticipated behavioral discrepancy, which needs further examinations in future studies. Also, the concept and measurement of anticipated behavioral discrepancy should be further developed and validated. Third, stated behaviors are different from actual behaviors. However, in the context of examining nPEP as a potential national strategy for HIV prevention, the investigation of anticipated behavioral changes can provide valuable data for strategy planning and policy development. Fourth, qualitative studies exploring the whole process and in-depth experiences of using nPEP services from users' perspective are warranted, so that researchers can develop better knowledge of using nPEP services for health behavior promotion for the key population.

\section{Conclusions}

This study identified a high-risk subgroup of MSM who reported they would use condoms less and/or have more sexual partners when nPEP becomes available. These men were younger in age, had shorter duration of residence in the metropolitan city, and had higher education. Tailored health interventions might be needed to mitigate potential increases in sexual risk behaviors for this subgroup of MSM. Moreover, there is a clear discrepancy between anticipated individual and population behavioral changes regarding future routinization of nPEP services, suggesting the need to address the issue of distrust and internalized stigma among MSM population.

\section{Abbreviations \\ AIDS: Acquired immune deficiency syndrome; CBO: Community-based organization; CFA: Confirmatory factor analysis; CFI: Comparative fit index; Cl: Confidence interval; HIV: Human immunodeficiency virus; MSM: Men who have sex with men; nPEP: Non-occupational Post-exposure Prophylaxis; CYN: China Yuan; RMSEA: Root mean square error of approximation; SD: Standardized deviation; SEM: Structural equation modeling; \\ SPSS: Software package for statistical analysis; STI: Sexually transmitted infection; TLI: Tucker Lewis index; USD: United States dollar}

\section{Acknowledgements}

We appreciate the men who participated in this study and local CBOs helping the data collection. We also thank Canrui Jin and Liyan Wang in the People's Medical Publishing House who helped with the field work.

\section{Authors' contributions}

$H L$, RW, WM contributed to the conception and design of the study. $H L$ and JJO drafted the paper. WM provided oversight for data collection. HL, RW and $\mathrm{XN}$ assisted in the data collection. RW, HL, JJO, and TLW searched the literature. HL and ESK analyzed the data. TLW provided substantial comments and careful edit on drafts. All authors revised the manuscript and approved the final version to be published.

\section{Funding}

This work was supported by the National Key Research and Development Program (2017YFE0103800) and the National Science and Technology Major Project (2018ZX10721102-003-004) in China. The listed grant funders played no role in any step of this study. The content of the article is solely the responsibility of the authors and does not necessarily represent the official views of the funders.

\section{Availability of data and materials}

The datasets used and/or analyzed during the current study are available from the corresponding author on reasonable request. 


\section{Ethics approval and consent to participate}

This study was approved by the Ethics Review Committee of Public Health, Shandong University (IRB No:20180904). Written informed consent has been obtained from all participants.

\section{Consent for publication}

Not applicable.

\section{Competing interests}

The authors declare that they have no competing interests.

\section{Author details}

'Department of Epidemiology, School of Public Health, Cheeloo College of Medicine, Shandong University, 44 West Wenhua Road, Jinan 250012, Shandong Province, China. ${ }^{2}$ Monash University Central Clinical School, Melbourne, VIC 3004, Australia. ${ }^{3}$ Department of Educational and Psychological Studies, University of South Florida, Tampa, FL 33620-9951, USA. ${ }^{4}$ Department of Psychology, Rhode Island College, Providence, RI 02908-1924, USA. ${ }^{5}$ Peking University School of Pharmaceutical Sciences, Beijing 100191, China.

Received: 14 January 2020 Accepted: 20 January 2021

Published online: 26 January 2021

\section{References}

1. Centers for Disease Control and Prevention, \& U. S. Department of Human and Health Services. Updated Guidelines for Antiretroviral Postexposure Prophylaxis After Sexual, Injection Drug Use, or Other Nonoccupational Exposure to HIV-United States, 2016. [cited 2019 Nov 3]. Available from https://www.cdc.gov/hiv/pdf/programresources/cdc-hiv-npep-guidelines. pdf.

2. Centers for Disease Control and Prevention. Effectiveness of Prevention Strategies to Reduce the Risk of Acquiring or Transmitting HIV. 2017. [cited 2019 Nov 3]. Available from https://www.cdc.gov/hiv/risk/estimates/ preventionstrategies.html.

3. World Health Organization. Guidelines on post-exposure prophylaxis for HIV and the use of co-trimoxazole prophylaxis for HIV-related infections among adults, adolescents and children: Recommendations for a public health approach. 2014. [cited 2019 Dec 3]. Available from https://apps.who.int/iris/ bitstream/handle/10665/145719/9789241508193_eng.pdf;jsessionid=95884 E0BA394F739B5D92D056E5B912F? sequence=1.

4. Schechter M, do Lago RF, Mendelsohn AB, Moreira Rl, Moulton LH, Harrison $\mathrm{LH}$, et al. Behavioral impact, acceptability, and HIV incidence among homosexual men with access to postexposure chemoprophylaxis for HIV. J Acquir Immune Defic Syndr. 2004;35(5):519-25. https://doi.org/10.1097/ 00126334-200404150-00010.

5. Pinkerton SD, Martin JN, Roland ME, Katz MH, Coates TJ, Kahn JO. Costeffectiveness of HIV postexposure prophylaxis following sexual or injection drug exposure in 96 metropolitan areas in the United States. AIDS. 2004;18: 2065-73 [PubMed: 15577628].

6. Guinot D, Ho MT, Poynten IM, McAllister J, Pierce A, Pell C, et al. Costeffectiveness of HIV nonoccupational postexposure prophylaxis in Australia. HIV Med. 2009;10:199-208 [PubMed:19207598]

7. Jain S, Mayer KH. Practical guidance for nonoccupational postexposure prophylaxis to prevent HIV infection: an editorial review. AIDS. 2014;28(11): 1545-54. https://doi.org/10.1097/QAD.0000000000000301.

8. Leshin D, OlshtainPops K, Moses A, Elinav H. Limited awareness of the effective timing of HIV post-exposure prophylaxis among people with highrisk exposure to HIV. Eur J Clin Microbiol Infect Dis. 2019;38(4):779-84. https://doi.org/10.1007/s10096-019-03476-4.

9. Golub SA, Rosenthal L, Cohen DE, Mayer KH. Determinants of high-risk sexual behavior during post-exposure prophylaxis to prevent HIV infection. AIDS Behav. 2008;12(6):852-9. https://doi.org/10.1007/s10461-007-9286-8.

10. Heuker J, Sonder GJ, Stolte I, Geskus R, van den Hoek A. High HIV incidence among MSM prescribed postexposure prophylaxis, 2000-2009: indications for ongoing sexual risk behaviour. AIDS. 2012;26(4):505-12. https://doi.org/ 10.1097/QAD.0b013e32834f32d8.

11. Poynten IM, Jin F, Mao L, Prestage GP, Kippax SC, Kaldor JM, et al. Nonoccupational postexposure prophylaxis, subsequent risk behaviour and HIV incidence in a cohort of Australian homosexual men. AIDS. 2009;23(9): 1119-26. https://doi.org/10.1097/QAD.0b013e32832c1776.
12. Ong JJ, Landika A, Fairley CK, Bradshaw C, Chen M, Read TRH, et al. Characteristics, sexual practices and sexually transmissible infections diagnoses of men who have sex with men and use non-occupational HIV post-exposure prophylaxis in Victoria, Australia. Sex Health. 2016;13(6):555-9. https://doi.org/10.1071/sh16092.

13. Waldo CR, Stall RD, Coates TJ. Is offering post-exposure prevention for sexual exposures to HIV related to sexual risk behavior in gay men? AIDS. 2000;14(8):1035-9. https://doi.org/10.1097/00002030-200005260-00016.

14. Martin JN, Roland ME, Neilands TB, Krone MR, Bamberger JD, Kohn RP, et al. Use of postexposure prophylaxis against HIV infection following sexual exposure does not lead to increases in high-risk behavior. AIDS. 2004;18(5): 787-92.

15. Donnell D, Mimiaga MJ, Mayer K, Chesney M, Koblin B, Coates T. Use of non-occupational post-exposure prophylaxis does not lead to an increase in high risk sex behaviors in men who have sex with men participating in the EXPLORE trial. AIDS Behav. 2010;14(5):1182-9. https://doi.org/10.1007/ s10461-010-9712-1.

16. Zeng Z, Liu H, Xu J, Lan G, Wang L, Yan W. Demand for non-occupational post-exposure prophylaxis and its influenting factors among 344 men who have sex with men in Guangxi. Chin J AIDS STD. 2017;23(7):620-4.

17. Yang M, Yan H, Lun W, Liu Y, Wu Y, Xu K. Current situation of post-exposure HIV prophylaxis and its interference after non-occupational needle stick or accidental injuries. Chin J AIDS STD. 2005;11(5):342-4.

18. Bandura A. A social cognitive theory of personality. In: Pervin L, John O, editors. Handbook of personality. 2nd ed. New York: Guilford; 1999. p. 154-96.

19. Fernández-Balbuena S, Belza MJ, Castilla J, Hoyos J, Rosales-Statkus ME, Sanchez R, et al. Awareness and use of nonoccupational HIV post-exposure prophylaxis among people receiving rapid HIV testing in Spain. HIV Med. 2013;14:252-7. https://doi.org/10.1111/j.1468-1293.2012.01056.x.

20. Thomas PE, Voetsch AC, Song B, Calloway D, Goode C, Mundey L, et al. HIV risk behaviors and testing history in historically black college and university settings. Public Health Rep. 2008;123(Suppl 3):115-25. https://doi.org/10. 1177/00333549081230S314.

21. Conner M, McEachan R, Taylor N, O'Hara J, Lawton R. Role of affective attitudes and anticipated affective reactions in predicting health behaviors. Health Psychol. 2015;34(6):642-52. https://doi.org/10.1037/hea0000143.

22. Valshtein TJ, Seta CE. Behavior-goal consistency and the role of anticipated and retrospective regret in self-regulation. Motivation Sci. 2019;5(1):35-51. https://doi.org/10.1037/mot0000101.

23. Henny KD, Duke CC, Geter A, Gaul Z, Frazier C, Peterson J, et al. HIV-related training and corelates of knowledge, HIV screening and prescribing of NPEP and PrEP among primary care providers in Southeast United States, 2017. AIDS Behav. 2019;23(11):2926-35. https://doi.org/10.1007/s10461-019-02545-1.

24. Chomchey N, Woratanarat T, Hiransuthikul N, Lertmaharit S, Lohsoonthorn $V$, Teeratakulpisarn $N$, et al. Factors associated with intention to take nonoccupational HIV post-exposure prophylaxis among Thai men who have sex with men. J Virus Erad. 2017:3:128-39.

25. China Center for Disease Control and Prevention, \& Center for STDs and AIDS Control and Prevention. National HIV/AIDS sentinel surveillance implementation program-Operational manual. Beijing: China CDC; 2017.

26. Li H, Wei R, Piqueiras E, Chow EPF, Jiao K, Lewis T, et al. HIV nonoccupational postexposure prophylaxis (nPEP) usage among five key populations in China. Sex Transm Infect, 2021;0:1-3. doi:https://doi.org/10. 1136/sextrans-2020-054791.

27. Bentler PM. Comparative fit indexes in structural models. Psychol Bull. 1990; 107(2):238-46

28. Hu L, Bentler PM. Cutoff criteria for fit indexes in covariance structure analysis: conventional criteria versus new alternatives. Struct Equ Modeling. 1999;6(1):1-55.

29. Brown TA. Confirmatory factor analysis: for applied research. New York: Guilford Press; 2015.

30. Raykov T, Dimitrov DM, Asparouhov T. Evaluation of scale reliability with binary measures using latent variable modeling. Struct Equ Modeling. 2010; 17(2):265-79.

31. Gefen D, Straub D, Boudreau M-C. Structural equation modeling and regression: guidelines for research practice. Commun Assoc Information Syst. 2000;4:7. https://doi.org/10.17705/1CAIS.00407.

32. Kim S, Sturman E, Kim ES. Structural equation modeling (SEM): principles, processes, and practices. In: Strang KD, editor. Palgrave handbook of research design in business and management. New York: PalgraveMacmillan; 2015. p. 153-72. 
33. Kline RB. Principles and practice of structural equation modeling. 4th ed. New York: Guilford Press; 2016.

34. Beauducel A, Herzberg PY. On the performance of maximum likelihood versus means and variance adjusted weighted least squares estimation in CFA. Struct Equ Model. 2006;13(2):186-203. https://doi.org/10.1207/ s15328007sem1302_2.

35. Dolan CV. Factor analysis of variables with 2, 3, 5 and 7 response categories: a comparison of categorical variable estimators using simulated data. $\mathrm{Br} \mathrm{J}$ Math Stat Psychol. 1994;47(2):309-26. https://doi.org/10.1111/j.2044-8317. 1994.tb01039.x.

36. Rhemtulla M, Brosseau-Liard PE, Savalei V. When can categorical variables be treated as continuous? A comparison of robust continuous and categorical SEM estimation methods under suboptimal conditions. Psychol Methods. 2012;17(3):354-73. https://doi.org/10.1037/a0029315.

37. Newcomb ME. Moderating effect of age on the association between alcohol use and sexual risk in MSM: evidence for elevated risk among younger MSM. AIDS Behav. 2013;17(5):1746-54. https://doi.org/10.1007/ s10461-013-0470-8.

38. Outlaw AY, Hightow-Weidman LB, Fields SD, Hidalgo J, Halpern-Felsher B, Green-Jones M. Age of MSM sexual debut and risk factors: results from a multisite study of racial/ethnic minority YMSM living with HIV. AIDS Patient Care STDS. 2011;25 Suppl 1(8):S23-9.

39. van der Snoek EM, de Wit JBF, Mulder PGH, van der Meijden WI. Incidence of sexually transmitted diseases and HIV infection related to perceived HIV/ AIDS threat since highly active antiretroviral therapy availability in men who have sex with men. Sex Transm Dis. 2005;32(3):170-5. https://doi.org/10. 1097/01.olq.0000149786.09706.66.

40. Li H, Holroyd E, Lau J, Li X. Stigma, subsistence, intimacy, face, filial piety, and mental health problems among newly HIV-diagnosed men who have sex with men in China. J Assoc Nurses AIDS Care. 2015;26(4):454-63. https:// doi.org/10.1016/j.jana.2015.02.004

41. Li H. Secondary HIV transmissions via newly diagnosed HIV positive men who have sex with men (MSM) in Shenzhen, China - a qualitative study. Hong Kong SAR: The Chinese University of Hong Kong; 2012.

42. Li H, Holroyd E, Lau JTF. Negotiating homosexual identities: the experiences of men who have sex with men in Guangzhou. Cult Health Sex. 2010;12(4): 401-14.

43. Lau JT, Wang M, Wong HN, Tsui HY, Jia M, Cheng F, et al. Prevalence of bisexual behaviors among men who have sex with men (MSM) in China and associations between condom use in MSM and heterosexual behaviors. Sex Transm Dis. 2008;35(4):406-13. https://doi.org/10.1097/OLQ. Ob013e318164467f.

\section{Publisher's Note}

Springer Nature remains neutral with regard to jurisdictional claims in published maps and institutional affiliations.

Ready to submit your research? Choose BMC and benefit from:

- fast, convenient online submission

- thorough peer review by experienced researchers in your field

- rapid publication on acceptance

- support for research data, including large and complex data types

- gold Open Access which fosters wider collaboration and increased citations

- maximum visibility for your research: over $100 \mathrm{M}$ website views per year

At BMC, research is always in progress.

Learn more biomedcentral.com/submissions 